\title{
DEPRESSÃO NA ESCLEROSE MULTIPLA FORMA REMITENTE-RECORRENTE
}

\author{
Maria Fernanda Mendes', Charles Peter Tilbery², Silvia Balsimelli4, \\ Marcos Aurélio Moreira ${ }^{3}$, Ana Maria Barão-Cruz ${ }^{4}$
}

\begin{abstract}
RESUMO - A possibilidade de correlação entre depressão e esclerose múltipla (EM) é conhecida há muitos anos, porém os estudos de prevalência não são conclusivos. No nosso meio a prevalência deste sintoma na EM permanece desconhecida. $O$ objetivo deste estudo é verificar a prevalência da depressão em pacientes com EM, estudando a sua correlação com a incapacidade funcional, o sexo, a idade e o tempo de doença. Foram avaliados 84 pacientes com EM remitente-recorrente (EMRR). A depressão foi avaliada através da Escala de Beck e da Escala para Ansiedade e Depressão (HAD), e a incapacidade funcional pela Escala de Incapacidade Funcional Expandida (EDSS). A depressão estava presente em 17,9\% e a ansiedade em 34,5\% dos pacientes com EMRR. Os maiores escores das escalas de depressão correlacionaram-se com maior incapacidade funcional $(p=0,0002)$, porém não estão associados ao tempo de doença, ao sexo ou a idade dos pacientes. Nossos dados indicam que a depressão é frequente nos pacientes com EM e sugerem haver correlação entre a depressão e a incapacidade funcional.
\end{abstract}

PALAVRAS-CHAVE: depressão, esclerose múltipla, incapacidade funcional, EDSS.

\section{Depression in relapsing-remitting multiple sclerosis}

\begin{abstract}
The suggestion of a possible relationship between depression and multiple sclerosis (MS) has existed for many years, and the prevalence studies are believed by potential biases. In our country, the prevalence of clinical depression in patients with MS is unknown. The objective of the present study was to ascertain the rate of depression in a group of MS patients and to analyze the relationship to depression, disability, gender, age and duration of illness. We evaluated 84 relapsing-remitting MS patients using the Beck Scale (BS), the Hospital Anxiety and Depression scale (HAD) and the Expanded Disability Status Scale (EDSS). The depression was presented at $17.9 \%$ and the anxiety at $34.5 \%$ of the RRMS patients. There is a correlation between depression and functional disability $(p=0.0002)$, but there is no relation between depression and sex, age or duration of the illness. This analysis confirms that depression is common in persons with MS and suggests the association with functional disability .
\end{abstract}

KEY WORDS: depression, multiple sclerosis, disability, EDSS.

Alterações de humor, como euforia, depressão e estado de apatia nos pacientes com esclerose múltipla (EM) são conhecidas desde as primeiras descrições da doença, Charcot já as referindo como parte do quadro clínico de EM ${ }^{1}$. Desde então, diversos pesquisadores estudam a prevalência e a fisiopatologia destes sintomas na EM. Enquanto alguns apontam para uma alta prevalência destes, outros os consideram como sendo de menor importância. A diversidade dos estudos e das metodologias aplicadas faz com que, muitas vezes, os resultados não sejam com- paráveis, o que dificulta ainda mais a compreensão destas manifestações ${ }^{2}$.

A depressão é um dos sintomas emocionais que frequentemente encontra-se associada à EM. Os primeiros estudos sobre prevalência datam de 1950, porém os resultados são divergentes. Acomete de 27 a $54 \%$ dos pacientes, embora taxas mais baixas tenham sido estimadas, e os sintomas são descritos como moderados ou graves ${ }^{3}$. A taxa de suicídio não é bem determinada, e estudo realizado por Kahana et al. ${ }^{4} \mathrm{em} 1971$, com 295 pacientes a estimou em 3\%,

Centro de Atendimento e Tratamento de Esclerose Múltipla (CATEM) da Disciplina de Neurologia do Departamento de Medicina da Faculdade de Ciências Médicas da Santa Casa de São Paulo, São Paulo SP, Brasil: ${ }^{1}$ Professor Instrutor; ${ }^{2}$ Professor Adjunto; ${ }^{3}$ Pós-Graduando; 4 Psicóloga.

Recebido 13 Dezembro 2002, recebido na forma final 11 Março 2003. Aceito 21 Março 2003.

Dra. Maria Fernanda Mendes - Rua Dr. Martinico Prado 26/122 - 01224-010 São Paulo SP - Brasil. 
enquanto que, em um estudo multicêntrico realizado no Canadá, com 3125 pacientes, observou-se que a mesma é 7,5 vezes maior que a da população geral para a mesma idade ${ }^{5}$, e a ideação suicida está presente em $28,6 \%$ dos pacientes com $\mathrm{EM}^{6}$.

No Brasil, não existem dados sobre a prevalência da depressão na EM. O objetivo deste estudo é verificar a sua frequência nos paciente com EM remitente-recorrente e analisando a sua correlação com a duração da doença e incapacidade funcional.

\section{MÉTODO}

Foram avaliados 84 pacientes que ingressaram no Centro de Atendimento e Tratamento de Esclerose Múltipla (CATEM) da Disciplina de Neurologia da Faculdade de Ciências Médicas da Santa Casa de Misericórdia de São Paulo (FCMSCM-SP) com diagnóstico de EM, definida de acordo com os critérios clínicos de Poser e cols ${ }^{7}$, todos com a forma clínica remitente-recorrente da doença (EMRR), conforme a classificação adotada por Lublin \& Reingold ${ }^{8}$, independemente da queixa prévia de depressão. A pesquisa foi aprovada pelo Comitê de Ética Médica da FCMSCM-SP.

Foram excluídos do estudo pacientes com escolaridade inferior a 4 anos completos; uso de corticosteróides, benzodiazepínicos, carbamazepina, gabapentina ou outras drogas antidepressivas nos últimos 3 meses; uso de terapêutica imunomoduladora ou imunossupressora; antecedente de uso abusivo de drogas e álcool; pacientes que não aceitaram participar do estudo.

Todos os pacientes incluídos na avaliação foram examinados por médicos neurologistas quanto à incapacidade funcional, através da escala de Kurtzke ${ }^{9}$ (EDSS) e entrevistados por psicólogas quanto à depressão. Para quantificar este sintoma, foi aplicada a Escala de Ansiedade e Depressão ${ }^{10}$ (HAD) e da Escala de Beck ${ }^{11}$ para depressão.

A Escala de Beck é composta por 21 itens, sendo possível em cada um deles uma resposta cujo escore varia de 0 a 4 (ausente, leve, moderada e grave), permitindo quantificar a intensidade do sintoma. Esta escala avalia os diver- sos sintomas ou atitudes que usualmente estão presentes na depressão, independentemente da sua causa. Dentre estas questões, existem itens relacionados com humor, pessimismo, insatisfação, autocrítica, irritabilidade, alterações de peso, dificuldades para o trabalho, fadiga, preocupações com a saúde, alterações de libido, entre outras. 0 escore igual ou superior a 18 foi considerado indicativo da presença de depressão ${ }^{11}$.

A HAD é uma escala que foi desenvolvida para pacientes hospitalizados ou portadores de doenças crônicas, evitando perguntas que possam ser atribuídas tanto à depressão, quanto a sintomas da doença ${ }^{10}$, e validada para a língua portuguesa ${ }^{12}$. É um questionário composto por 14 itens, subdivididos em duas subescalas, sendo que sete avaliam o sintoma depressão (HAD-d) e sete o sintoma ansiedade (HAD-a). Consideramos o escore igual ou superior a oito como indicativo de ansiedade ou depressão, por ser este o escore mais sensível para detectar depressão ou ansiedade ${ }^{10}$.

As duas escalas foram aplicadas em 84 pacientes. 0 grupo com depressão foi formado por pacientes com escore igual ou superior a 18 na Escala de Beck e/ou oito na HAD. Os pacientes sem alterações em ambas as escalas foram incluídos no grupo sem depressão. Os grupos com e sem depressão foram comparados quanto às variáveis de sexo, idade, tempo de duração da doença e grau de incapacidade funcional pelo Teste t-Student. $O$ teste de correlação de Spearman foi utilizado para verificar a associação com a incapacidade funcional, o tempo de doença, a ansiedade e a correspondência entre os escores obtidos nas duas escalas.

Os escores obtidos nas duas escalas foram classificados quanto à presença ou ausência da depressão e os resultados obtidos pelos diferentes instrumentos foram confrontados com a aplicação do teste do qui-quadrado.

\section{RESULTADOS}

O grupo foi composto por 71 mulheres $(84,5 \%)$ e 13 homens (15,5\%), com faixa etária compreendi-

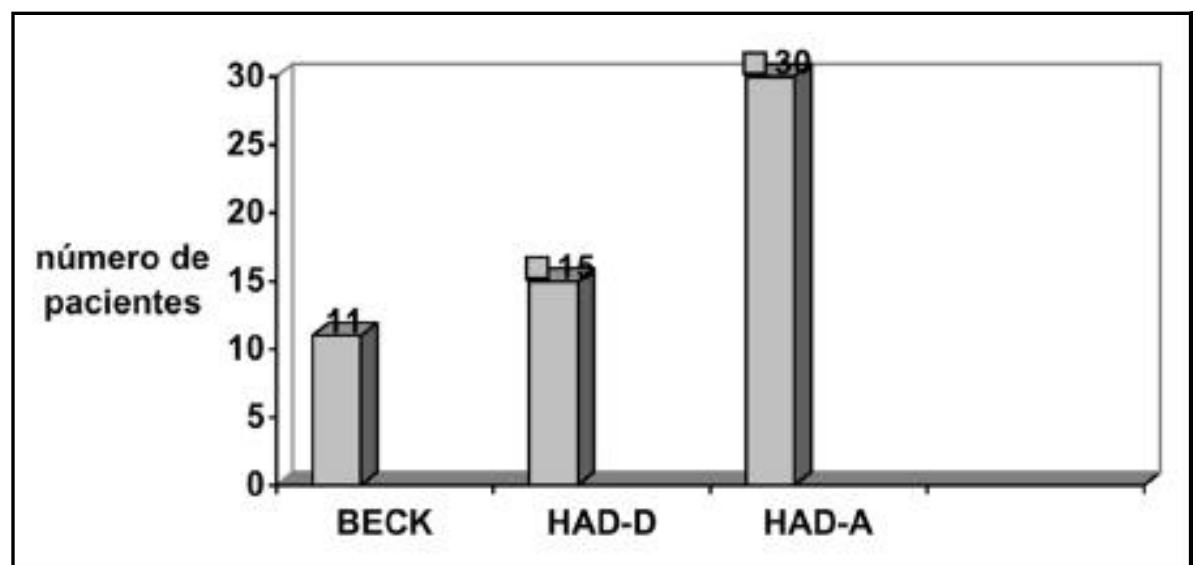

Fig 1. Gráfico de presença de ansiedade e depressão nas escalas de Beck ou na escala de ansiedade de depressão (HAD) nos pacientes com esclerose múltipla remitente-recorrente. 
Tabela 1. Comparação entre os resultados obtidos pelos pacientes com esclerose múltipla nas escalas de Beck e escala de Ansiedade e Depressão (HAD).

\begin{tabular}{lcccc}
\hline & \multicolumn{2}{c}{ Depressão } & & Ansiedade \\
\cline { 2 - 3 } & Beck & HAD-D & & HAD-A \\
\hline Presente & $11(13,1 \%)$ & $15(17,9 \%)$ & & $30(35,7 \%)$ \\
Ausente & $73(86,9 \%)$ & $69(82,1 \%)$ & & $54(64,3 \%)$ \\
\hline
\end{tabular}

*sem diferenças estatisticamente significantes entre os grupos pelo Teste do Qui-Quadrado. HAD-D, subescala depressão; HAD-A, subescala ansiedade na escala de ansiedade e depressão.

Tabela 2. Características dos pacientes com e sem depressão quanto as variáveis de sexo, idade, duração da doença e incapacidade funcional.

\begin{tabular}{lcc}
\hline & $\begin{array}{c}\text { Com depressão } \\
(\mathrm{n}=19)\end{array}$ & $\begin{array}{c}\text { Sem depressão } \\
(\mathrm{n}=65)\end{array}$ \\
\hline $\begin{array}{l}\text { Sexo } \\
\text { Feminino }\end{array}$ & $15(78,9 \%)^{\#}$ & $56(86,2 \%)$ \\
Masculino & $4(21,1 \%)$ & $9(13,8 \%)$ \\
Idade (anos) & $36,1(8,9)^{\S}$ & $34,1(8,8)$ \\
Média (Desvio Padrão) & 35 & 36 \\
Mediana & $21-54$ & $18-48$ \\
Faixa etária & & \\
Tempo de doença (meses) & $73,7(47,9)^{* *}$ & $46,7(32,8)$ \\
Média (desvio padrão) & 60 & 42 \\
Mediana & & $0-6$ \\
EDSS & $3,1(1,5)^{*}$ & 1,5 \\
Média (desvio padrão) & 3,5 & $0-3,5$ \\
Mediana & $0-6$ & \\
Faixa do EDSS &
\end{tabular}

* com diferença estatisticamente significante pelo teste t-Student para $p=0,001 ; \# p=0,3460$ pelo teste de Fischer; $\S p=0,3450$ pelo teste tStudent; ** $p=0,0716$ pelo teste $t$-Student. P, significância; $F$, feminino; $M$, masculino; $n$, número de sujeitos, EDSS, escala de incapacidade funcional expandida.

da entre 17 e 50 anos, com média de idade de 34,6 $\pm(8,7)$ anos. A duração média da doença era de 57,8 meses $\pm(48,1)$, variando de 2 a 180 meses. $O$ escore do EDSS encontrava-se entre 0 e 6,0 , com média de $2,1 \pm(1,4)$.

Ambas as escalas foram aplicadas nos 84 pacientes, sendo que $30(35,7 \%)$ deles apresentaram alterações em pelo menos uma das escalas. De acordo com os escores obtidos na escala de Beck, a depressão foi diagnosticada em 11 pacientes (13,1\%). Na escala HAD sub item depressão, este sintoma foi detectado em 15 pacientes (17,9\%). A ansiedade foi detectada em 30 pacientes (35,7\%) com a aplicação da escala HAD sub item ansiedade (Fig 1).
Ao confrontarmos os resultados obtidos na escala de Beck com aquele obtido na HAD, nenhuma diferença estatisticamente significante foi observada quanto ao número de pacientes com depressão (Tabela 1). Todos os pacientes com escores para depressão na escala de Beck apresentaram alterações também na HAD, sendo que em 7 deles observamos depressão e ansiedade, e em quatro somente ansiedade (Quadro 1). Estes apresentavam escores baixos na escala de Beck (18, 20,21 e 23), e em dois deles o escore para ansiedade foi inferior a 11 (9 e 10).

Ao compararmos as principais características dos dois grupos, não observamos diferenças quanto ao sexo, a idade e o tempo de doença. O EDSS médio dos pacientes sem depressão era de $1,50(0,84)$ e dos pacientes com depressão $3,18(1,49)$, diferença esta que foi estatisticamente significante $(p=0,0002)$ (Tabela 2).

Ao realizarmos a análise de correlação de Spearman observamos que os escores obtidos nas escalas HAD-d e Beck encontram-se correlacionados $(p<0,001)$, assim como os escores da HAD-a $(p<0,001)$. Nenhuma correlação foi observada com a duração da doença $(p=0,086)$, porém esta foi observada com a incapacidade funcional, mensurada pelo EDSS $(p=0,0002)$.

\section{DISCUSSÃO}

Os estudos sobre a depressão na EM são escassos e a sua prevalência não é determinada. Estudos estimam que este sintoma acomete de 27 a $54 \%$ dos pacientes durante o curso da doença ${ }^{3,13}$. Na nossa série observamos uma menor prevalência deste sintoma, que variou de $13,1 \%$ a $17,9 \%$, de acordo com o teste utilizado, sendo que a nossa série se diferencia das demais pois apenas pacientes com a EMRR foram avaliados. A exclusão de pacientes com as formas progressivas da doença, e, consequentemente, aqueles com maior incapacidade, pode ter interferido nestes resultados. Com relação à ansiedade, existem raros estudos na literatura, embora ela aparente-

Quadro 1. Distribuição dos pacientes nas escalas de BECK e subitens da escala de ansiedade e depressão.

\begin{tabular}{lc}
\hline Escalas & Pacientes (n) \\
\hline HAD-A & 14 \\
HAD-D & 1 \\
HAD-A + HAD-D & 4 \\
HAD-A + HAD-D + Beck & 7 \\
HAD-A + Beck & 4 \\
\hline
\end{tabular}


mente encontre-se associada à presença de ideação suicida ${ }^{6}$. Nossos achados foram semelhantes àqueles obtidos Feinstein ${ }^{6}$, que estimou a prevalência deste sintoma em $35,7 \%$ dos pacientes com EM.

Não é referida correlação entre a depressão e o tipo ou intensidade do déficit neurológico, a forma clínica da doença, a duração dos sintomas ou presença de alterações cognitivas, embora a depressão seja mais frequente nos períodos de exacerbação da doença ${ }^{2,14}$. Neste estudo, observamos que o sexo, a idade e o tempo de doença não interferem no aparecimento da depressão, porém a incapacidade funcional associa-se com o aparecimento deste sintoma, sendo encontrada correlação entre a intensidade do déficit neurológico com os escores mais elevados das escalas de depressão. Outros autores não observaram esta correlação $0^{3,15}$, porém ressaltamos novamente que o nosso grupo era constituído exclusivamente por pacientes com EMRR e incapacidade funcional de leve a moderada.

Rabins et al. ${ }^{16}$, em 1986, observaram que os pacientes com EM são mais deprimidos que os lesados medulares, embora estes apresentem um grau maior de incapacidade motora. Porém, entre os pacientes com EM, aqueles com lesão cerebral apresentavam escores maiores para depressão que aqueles com lesão predominante medular, não observando correlação com incapacidade, sugerindo que a depressão é resultante da associação entre fatores psicológicos e a presença de lesões cerebrais.

Ainda hoje, especula-se se a depressão é uma forma de manifestação clínica de um processo desmielinizante ativo em região subcortical, ou se há apenas uma coincidência ou ainda associação de fatores ${ }^{17}$. Nossos achados sugerem haver a predominância de uma base orgânica para explicar o aparecimento da depressão, pois no grupo estudado a incapacidade funcional era leve. A interrupção das vias cortico-subcorticais secundárias às lesões desmielinizantes talvez explique a correlação entre a intensidade da depressão e da incapacidade funcional, porém estudos de neuroimagem realizados de forma sistemática nestes pacientes são necessários para melhor elucidação dos achados.

É relatada a correlação entre lesões desmielinizantes periventriculares e distúrbios do humor ${ }^{2}$. Recentemente, observou-se que pacientes com EM que apresentavam sintomas de depressão apresentavam lesões predominantemente em lobo temporal direito, o que não era observado naqueles não deprimi$\operatorname{dos}^{15}$. A associação entre a intensidade da depres- são e a presença de lesões hipointensas em $T_{1}$ em região frontal superior e parietal superior e temporal, e atrofia frontal também foi relatada ${ }^{18}$, sugerindo que a depressão na EM pode ser secundária a desconexão cortico subcotical, com interrupção das vias de projeção do sistema límbico basal.

Autores que buscam uma explicação psicodinâmica sugerem que os sintomas depressivos não resultam da atividade da doença, e sim do medo e apreensão frente ao diagnóstico e das perdas que advém da evolução da doença ${ }^{3}$. A associação entre a ansiedade e a depressão observada nos pacientes aqui estudados aparentemente corrobora a teoria psicodinâmica, porém no nosso estudo a duração média da doença era de apenas quatro anos, o que pode ter interferido nestes achados. Embora existam evidências nos dois sentidos e os estudos não sejam conclusivos, acreditamos haver uma base multifatorial para a manifestação deste sintoma, com o envolvimento de fatores biológicos, psicológicos e genéticos.

Embora o tratamento da depressão na EM não seja específico, a avaliação destes sintomas nos portadores de EM é fundamental para completa abordagem destes pacientes. Na prática clínica é difícil definir qual o melhor método para a avaliação dos pacientes. A aplicação de escalas de fácil aplicação e compreensão, rápidas e cujos itens não se confundam com a doença de base dos pacientes é fundamental. Nos pacientes com EM, a depressão assume características peculiares, confundindo-se com a sensação de fadiga e com as alterações cognitivas. Várias escalas vêm sendo utilizadas para a mensuração deste sintoma, porém não há consenso quanto a melhor escala a ser utilizada. $\mathrm{O}$ uso de diferentes instrumentos para a medida da depressão é um dos fatores que dificulta a análise da frequência, da intensidade e da etiologia deste sintoma.

Ambas as escalas aqui aplicadas foram bem aceitas pelos pacientes, facilmente compreendidas e mostraram-se eficazes para a avaliação da depressão na EM. A Escala de Beck é muito utilizada, porém, inclui entre os itens perguntados sintomas que podem estar correlacionados à EM e não à depressão, como fadiga e interesse sexual, entre outros ${ }^{11,19}$. A escala HAD não inclui perguntas que possam ser confundidas com os sintomas somáticos associados às alterações do humor. Esta escala demonstrou ser um instrumento mais eficiente para o screening de depressão e ansiedade nos pacientes com esclerose múltipla, por proporcionar uma avaliação mais abrangente do estado emocional do paciente. 


\section{CONCLUSÃO}

1. A depressão está presente em $17,9 \%$ e a ansiedade em $35,7 \%$ dos pacientes com EM forma remitente recorrente.

2. Os maiores escores das escalas de depressão correlacionam-se com maior incapacidade funcional, porém não estão associados ao tempo de doença.

3. A escala HAD é um instrumento adequado para a avaliação da depressão na EM.

\section{REFERÊNCIAS}

1. Murray TJ. The history of multiple sclerosis. In Burkes JS, Johnson KP. (eds) Multiple sclerosis: diagnosis, medical management and rehabilitation. New York: Demos Medical, 2000:1-32.

2. Minden SL, Schiffer RB. Depression and affective disorders in multiple sclerosis. In Halbreich U. (ED) Multiple sclerosis: a neuropsychiatric disorder. Washington: American Psychiatric Press; 1993:33-54.

3. Minden SL, Schiffer RB. Affective disorders in multiple sclerosis: review and recommendations for clinical research. Arch Neurol 1990;47:98-104.

4. Sadovnick AD, Eisen K, Patty DW, Ebers GC. Cause of death in patients attending multiple sclerosis clinics. Neurology 1991;41:1193-1196.

5. Kahana E, Leibowitz U, Alter M. Cerebral multiple sclerosis. Neurology 1971;21: 1179-1185.

6. Feinstein A. An examination of suicidal intent in patients with multiple sclerosis. Neurology 2002;59:674-678.
7. Poser CM, Paty DW, Scheinber GL, et al. New diagnostic criteria for multiple sclerosis: guidelines for research protocols. Ann Neurol 1993;13:227-231.

8. Lublin FD, Reingold SC. Defining the clinical course of multiple sclerosis: results of an international survey. Neurology 1996;46:907-911.

9. Kurtzke JF. Rating neurologic impairment in multiple sclerosis: an expanded disability status scale (EDSS). Neurology 1983;33:1444-1452.

10. Zigmond AS, Snalth RP. The Hospital Anxiety and Depression Scale. Acta Psychiatr Scand 1983;67:361-370.

11. Beck AT, Ward CH, Mendelson M, Mock J, Erbaugh J. An inventory for measuring depression. Arch Gen Psychiatry 1961;4:561-571.

12. Botega NJ, Bio MR, Zomignani MA, Garcia Junior C, Pereira W. Transtornos do humor em enfermaria de clínica médica e validação da escala de medida (HAD) de ansiedade e depressão. Rev Saúde Pública 1995;29:355-363.

13. Staneger EN, Staneger E, Henrikson NK, et al. Suicide and multiple sclerosis: an epidemiological investigation. J Neurol Neurosurg Psychiatry 1992;55:869-876.

14. Sadovnick AD, Remick RA, Allen J, et al. Depression and multiple sclerosis. Neurology 1996;46:628-632.

15. Berg D, Supprian T, Thomae J, et al. Lesion pattern in patients with multiple sclerosis and depression. Mult Scler, 2000;6:156-162.

16. Rabins PV, Brooks BR, O'Donnell P, et al. Structural brain correlates of emotional disorder in multiple sclerosis. Brain 1986;109:585-597.

17. Berrios GE, Quemada JI. Depressive illness in multiple sclerosis: clinical and theoretical aspects of the association. Br J Psychiatry 1990;156:10-16.

18. Bakshi R, Shaikh ZA, Miletich RS et al. Fatigue in multiple sclerosis and its relationship to depression and neurologic disability. Mult Scler 2000;6:181-185.

19. Aikens JE, Reinecke MA, Pliskin NH, et al. Assessing depressive symptoms in multiple sclerosis: is it necessary to omit items from the original Beck Depression Inventory? J Behav Med 1999;22:127-142. 